\title{
Desempenho em leitura/escrita e em cálculos aritméticos em crianças de $2^{\mathrm{a}}$ série
}

\author{
Clarissa Seligman Golbert \\ Jerusa Fumagalli de Salles
}

\begin{abstract}
Resumo
Esta pesquisa investiga os desempenhos em leitura e escrita e em aritmética básica. Dois grupos de crianças - 15 competentes em leitura/ escrita e 12 com dificuldades - foram comparados quanto ao desempenho em cálculos aritméticos com o intuito de identificar em que aspectos do desempenho numérico se assemelham ou se diferenciam. Investigou-se também a relação entre desempenho aritmético, tarefas neuropsicológicas (leitura, escrita, habilidades perceptivo-motoras, linguagem oral, velocidade de processamento, consciência fonológica, memória verbal, memória não verbal) e inteligência. Os resultados indicaram heterogeneidade de desempenhos intragrupos nas habilidades aritméticas. No grupo competente em leitura/escrita, foram identificadas algumas crianças com sérias dificuldades na aritmética, apresentando defasagens no senso numérico, nos procedimentos de contagem e nos fatos básicos da adição e subtração. Inversamente, no grupo com dificuldade em leitura/escrita, foram também encontradas crianças competentes na aritmética. A análise dos diferentes perfis dos subgrupos sugere a necessidade de recursos preventivos e terapêuticos diferenciados.
\end{abstract}

Palavras-chave: Matemática, leitura, escrita, consciência fonológica.

\section{Reading/Writing and arithmetic calculations performance in second-grade children}

\begin{abstract}
This research investigated the reading and writing disabilities and the Arithmetic learning disabilities. Two groups - one with 15 children proficient in reading and writing, and the other with 12 children with reading and writing disabilities - were compared in relation to the arithmetic processing. The aim was to identify in which aspects of numerical performance they were similar or different. It was also investigated the relation among arithmetic performance, neuropsychological tasks (reading, writing, perceptual-motor skills, oral language, processing speed, phonological awareness, verbal memory, and non-verbal memory), and intelligence. The results showed intra-group performance heterogeneity. In the reading and writing proficient group, children with severe difficulties in aritmetic were identified. Inversely, in the group with children with reading and writing disabilities, children proficient in arithmetic were found. The analysis of each subgroup according to the neuropsychological profile suggested that both preventive and therapeutic resources are necessary.
\end{abstract}

Keywords: Mathemathics, reading, writing, phonological awareness.

\section{Rendimiento en lectura/escritura y en cálculos aritméticos en niños de $2^{\circ}$ grado}

\section{Resumen}

Este estudio investiga los rendimientos en lectura/escritura y en aritmética básica. Se compararon dos grupos de niños - 15 competentes en lectura/escritura y 12 con dificultades en lectura/escritura - respecto a su rendimiento en cálculos aritméticos, con la intención de identificar en qué aspectos del rendimiento numérico se parecen o se diferencian. Se investigó también la relación entre rendimiento aritmético, tareas neuropsicológicas (lectura, escritura, habilidades perceptivo-motoras, lenguaje oral, velocidad de procesamiento, conciencia fonológica, memoria verbal, memoria no-verbal) e inteligencia. Los resultados indicaron heterogeneidad de rendimientos intragrupos en las habilidades aritméticas. En el grupo competente en lectura/escritura se identificaron algunos niños con serias dificultades en aritmética, presentando discrepancias en el sentido numérico, en los procedimientos de conteo y en las operaciones básicas de adición y substracción. Inversamente, en el grupo con dificultad en lectura/escritura también se encontraron niños competentes en aritmética. El análisis de los diferentes perfiles de los subgrupos sugiere la necesidad de recursos preventivos y terapéuticos diferenciados.

Palabras-clave: Matemática, lectura, escritura manual, consciencia fonológica. 


\section{Introdução}

O estudo e a busca de soluções para os problemas de aprendizagem aritmética passam pela construção de parâmetros que permitam análises consistentes de problemas tão complexos. É dentro dessa temática que se insere esta pesquisa. Compararam-se dois grupos de crianças competentes em leitura e escrita e com dificuldades - em relação ao desempenho aritmético, no intuito de identificar associações e dissociações ${ }^{1}$ entre desempenho em leitura e escrita e desempenho aritmético. Neste estudo, definese grupo competente em leitura e escrita aquele formado por crianças que apresentaram desempenho em tarefas de leitura e escrita superior à média dos colegas. O termo dificuldades de leitura e escrita inclui crianças com déficits na leitura e na escrita de palavras e de texto (Salles \& Parente, 2006).

A investigação das dificuldades de aprendizagem, em especial na leitura e escrita, teve grande impulso a partir da metade do século passado. Mais recentemente, em torno dos anos 80 , verificou-se um grande interesse pela cognição aritmética e pelos fatores cognitivos, pedagógicos e sociais que determinam o sucesso ou o fracasso nessa área (Berch \& Mazzocco, 2007; Fuchs \& Fuchs, 2002; Geary, 2004). A preocupação com os problemas na aritmética é plenamente justificada, levando-se em conta a importância destas habilidades básicas para o funcionamento produtivo na sociedade, fortemente influenciada pelo progresso tecnológico. Em lugar da fluência em cálculos, faz-se necessário um elaborado raciocínio matemático para interpretar informações numéricas cada vez mais sofisticadas (Geary, 2006; Gersten, Clarke \& Mazzocco, 2007; Jordan, 2007).

As estatísticas fornecidas pela UNESCO (Delors, 1999) em torno dos resultados da aprendizagem da matemática, não só no Brasil, indicam que um número expressivo de jovens, ao final do Ensino Médio, não apresenta a compreensão e os procedimentos numéricos requeridos pelo mercado de trabalho, como, por exemplo, conhecimento das propriedades do conjunto dos números naturais, das suas operações e das propriedades do sistema numérico decimal. É evidente que um atraso tão massivo tem implicações relativas ao modelo de ensino da matemática, refletindo lacunas do sistema educacional brasileiro. O relatório do INEP sobre o PISA - Programa Internacional de Avaliação de Alunos -, realizado em 2006, reforça este panorama: 44,5\% dos estudantes brasileiros executam apenas tarefas básicas de leitura, como localizar informação direta e compreender partes do texto, por exemplo, e em aritmética apresentam apenas habilidades numéricas básicas, isto é, podem empregar algoritmos e interpretar literalmente os resultados (www.inep.gov.br).

1 Associações de sintomas (síndromes) ocorrem quando há déficits de desempenho no mesmo conjunto de tarefas. Dissociações ocorrem por exemplo quando um caso "A" apresenta um desempenho satisfatório na tarefa 1 e um desempenho insatisfatório na tarefa 2 , mas um caso "B" apresenta um desempenho insatisfatório na tarefa 1 e satisfatório na tarefa 2 (Kristensen, Almeida, \& Gomes, 2001).
As dificuldades de leitura e escrita não deixaram de ser alvo de pesquisas e debates na literatura nacional (Kleiman, 2001; Salles, 2005; Salles \& Parente, 2002a, 2002b, 2006, 2008; Lins e Silva \& Spinillo, 1998; Pinheiro, 2001). Adicionalmente, com o avanço das pesquisas, as especificidades da aritmética foram sendo também reconhecidas e lança-se nova luz sobre as questões de avaliação, de ensino, de reabilitação e de prevenção das dificuldades na aprendizagem da aritmética. Na avaliação destas dificuldades, é fundamental discriminar quando se trata de uma dificuldade natural, que pode ser superada mais ou menos rapidamente, ou quando se trata de uma dificuldade secundária a outros transtornos, tais como problemas sensoriais, neurológicos, emocionais, sociais e/ou educacionais (Moojen \& Costa, 2006).

O transtorno na matemática, definido no DSM-IV-TR (2003) e universalmente aceito, é caracterizado como uma capacidade para a realização de operações aritméticas (medida por testes padronizados, individualmente administrados de cálculo e raciocínio matemático) acentuadamente abaixo da esperada para a idade cronológica, a inteligência medida e a escolaridade do indivíduo. Nessa condição, encontramse apenas $3 \%$ a $5 \%$ das crianças avaliadas em diferentes culturas e sistemas de ensino (Geary, 2007).

As crianças com dificuldade nessa área não formam um grupo homogêneo, pois o conhecimento matemático é constituído de vários componentes. Na visão de Mazzocco (2007), os obstáculos a essa compreensão têm origem tanto na complexidade da matemática (como, por exemplo, na integração de diferentes sistemas simbólicos) quanto nos seus correlatos cognitivos: linguagem, atenção, percepção visuoespacial, memória de trabalho, memória de longo prazo, enfim, o funcionamento neuropsicológico como um todo. Os pesquisadores ainda discordam quanto à terminologia, origem e critérios de avaliação das dificuldades na aritmética (Costa, 2007). Entretanto, apesar das diferenças teóricas e metodológicas, já há concordância de que a aritmética escolar tem seus alicerces em habilidades cognitivas básicas, linguísticas e perceptivas (Berch \& Mazzocco, 2007; Dehane \& Cohen, 1995; Durant, Hulme, Larkin \& Snowling, 2005).

Para Geary (2006), a maior parte dos problemas na aprendizagem da aritmética decorre de falhas nas noções fundamentais de quantidade, grandeza e extensão. Essas noções, construídas dos dois aos seis anos de idade, aproximadamente, resultam do desenvolvimento perceptivo, linguístico e de fatores culturais, e incidem sobre a elaboração da noção de número, que, por sua vez, sustenta todas as aquisições quantitativas e lógicas posteriores (Berch \& Mazzocco, 2007; Geary, 2006; Fuchs e cols., 2005). Frequentemente, na escola, essas habilidades não são reconhecidas, nem valorizadas, e as crianças são introduzidas nos cálculos mecanicamente.

Os estudos têm apontado três características principais, que independem de diferenças culturais e curriculares, encontradas nas crianças com problemas na aritmética (Dowker, 2004; Geary, 1993, 2004; Orrantia, 2006): 1) dificuldades na memória de longo prazo (armazenamento e 
recuperação dos fatos básicos); 2) compreensão imatura dos princípios da contagem; e 3) dificuldades na representação visuoespacial da informação quantitativa, incidindo na elaboração do senso numérico. Além disso, as crianças confundem numerais com escritas semelhantes, alteram a direção na resolução de algoritmos e falham nas tarefas que requerem o manejo da reta numérica.

O consenso entre os pesquisadores sobre as habilidades numéricas que são deficitárias nas crianças com dificuldade nessa área não se verifica em relação ao funcionamento neuropsicológico. Geary (2004), Fuchs e cols. (2005) e Jordan, Kaplan e Hanich (2002) concordam que podem ocorrer falhas em processos atencionais, dificuldades visuoespaciais, deficiências no processamento auditivo, lentidão de processamento e deficiências na memória de trabalho (Berch \& Mazzocco, 2007).

De todos esses processos, o que mais tem sido pesquisado é a memória de trabalho ou memória operacional, cujo modelo proposto por Baddeley (1996) define como a capacidade humana de manter informações da memória imediata, estocadas temporariamente, durante uma tarefa cognitiva, tal como a leitura de um texto ou a resolução de um problema matemático, por exemplo. Ao longo dos anos, Baddeley tem feito reformulações do seu modelo inicial, constituído, atualmente, de quatro componentes: 1) um controlador da atenção, o executivo central; 2) um componente fonológico, que armazena e manipula as informações baseadas na fala; 3) um componente visuoespacial, que armazena e manipula as informações visuoespaciais; e 4) um componente episódico, que integra as informações dos componentes verbais e visuoespaciais (Baddeley, 2000). Os quatro componentes da memória de trabalho são fundamentais na aprendizagem da matemática (Baddeley \& Andrade, 2000) e deficiências na memória de trabalho comprometem a representação e manipulação de informações nos sistemas de linguagem e visuoespaciais (Geary, Hamson \& Hoard, 2000).

Considerando os estudos sobre as habilidades de leitura e matemática, Geary (2004), Geary e cols. (2000), Fuchs e cols. (2005), Fuchs e cols. (2006), Jordan (2007) e Jordan e Hanich (2003) compararam grupos de crianças com dificuldade na matemática, com e sem dificuldades na leitura. Jordan, Hanich e Kaplan (2003) identificaram diferentes perfis funcionais de crianças: 1) com dificuldade na leitura e na matemática (DL/DM); 2) com dificuldade somente na matemática (DM) e 3) com dificuldade somente na leitura (DL). O grupo com dificuldade na leitura e na aritmética (DL/DM) e o grupo com dificuldade apenas na matemática (DM) têm em comum deficiências essenciais no senso numérico, incluindo processamento numérico e aritmética básica. Crianças com DM específicas, sem DL, apresentam dificuldades na noção de número, nos procedimentos de contagem, nas operações aritméticas e na fluência computacional, sendo que o grupo DL/DM apresenta desempenho mais comprometido do que o grupo DM, pois os alunos têm entraves tanto nas habilidades verbais quanto nas não verbais - percepção da magnitude dos números e construção de uma linha numérica mental, por exemplo, relacionadas com a aprendizagem da matemática (Geary 2004; Geary e cols., 2000; Fuchs e cols., 2006, Jordan, 2007).

Pesquisadores de diferentes concepções teóricas concordam que as operações numéricas iniciais, como, por exemplo, a compreensão do significado dos números e o desenvolvimento de estratégias de contagem até a resolução de problemas complexos se fundamentam no senso numérico.

Além de comparar dois grupos - competentes e com dificuldades em leitura/escrita - quanto ao desempenho em cálculos aritméticos, o presente estudo investigou a relação entre desempenho aritmético, tarefas neuropsicológicas (leitura, escrita, habilidades perceptivo-motoras, linguagem oral, velocidade de processamento, consciência fonológica, memória verbal, memória não verbal) e inteligência.

\section{Método}

\section{Participantes}

Os participantes foram dois grupos de crianças selecionados de uma amostra de 110 crianças de $2^{a}$ série do Ensino Fundamental de cinco escolas públicas estaduais : 1) Grupo de $2^{a}$ série com dificuldades de leitura e escrita ( $n=12 ; 07$ meninos e 05 meninas, com média de idade de 8,43 anos e cujo desempenho em quatro tarefas de leitura e escrita foi mais do que um (1) desvio-padrão abaixo da média); 2) Grupo de $2^{a}$ série competente em leitura e escrita ( $n=15 ; 06$ meninos e 09 meninas, com média de idade de 8,21 e com desempenho em quatro tarefas de leitura e escrita superior a um (1) desvio-padrão em relação à média).

Para a seleção da amostra, foi utilizado o desempenho em quatro tarefas: leitura de palavras isoladas, compreensão textual, escrita de palavras isoladas e produção textual escrita (Salles \& Parente, 2007, 2008). Aqueles casos que apresentaram valores superiores a um desvio padrão da média foram enquadrados no grupo competente em leitura e escrita, enquanto aqueles que apresentaram valores inferiores a menos um desvio padrão fizeram parte do grupo com dificuldades de leitura/escrita. Foram critérios de exclusão da amostra: 1) suspeita de déficit auditivo e/ou visual não corrigidos; 2) histórico de problemas neurológicos adquiridos; 3) portadores de necessidades educativas especiais, em regime de inclusão; e 4) desempenho no teste de Matrizes Progressivas Coloridas de Raven igual ou inferior a $25 \%$ ("intelectualmente deficiente" ou "definidamente abaixo da média").

\section{Procedimentos e instrumentos}

Foi comparado o desempenho em cálculos aritméticos entre os dois grupos de crianças. Posteriormente, a correlação entre os desempenho em cálculos aritméticos e outras habilidades neuropsicológicas foi analisada. A leitura de palavras isoladas (Salles, 2001; Salles \& Parente, 
2002a, 2002b) foi avaliada por 60 palavras que variam em suas características psicolinguísticas quanto à regularidade, lexicalidade, extensão e frequência de ocorrência na língua. A compreensão da leitura textual (Salles, 2001; Salles \& Parente, 2002b) foi avaliada de duas formas: 1) leitura silenciosa seguida de reprodução oral, 2) resposta a dez questões de múltipla escolha sobre a história lida.

A escrita de palavras isoladas mediante ditado, tarefa aplicada coletivamente, foi retirada do International Dyslexia Test (Capovilla, Smythe, Capovilla \& Everatt., 2001; Smythe \& Cappellini, 2008; Smythe \& Everatt, 2000) e compõe-se de 40 palavras. Por fim, as crianças foram solicitadas a escrever uma história, a partir de uma sequência de gravuras, e as histórias foram enquadradas em categorias, de acordo com os trabalhos de Brandão e Spinillo (2001), Lins e Silva e Spinillo (1998, 2000), Spinillo (2001).

O desempenho em cálculos aritméticos foi avaliado através do subteste de cálculos aritméticos do International Dyslexia Test - IDT (Capovilla e cols., 2001; Smythe \& Cappellini, 2008; Smythe \& Everatt, 2000). Este subteste incluía três adições de números de um algarismo $(7+8 ; 8$ $+4 ; 3+7$ ) e uma adição de números com dois algarismos, com transporte $(23+48)$; uma subtração de números de dois algarismos $(17$ - 8) e duas subtrações com retorno, incluindo dezena e centena (63 - 17, 106 - 19). A multiplicação foi avaliada através de três cálculos com números de dois algarismos (3 $\times 8 ; 5 \times 6 ; 2 \times 8)$.

As funções neuropsicológicas - habilidades perceptivo-motoras, velocidade de processamento, consciência fonológica, memória verbal e memória não-verbal - foram, em geral, retiradas do International Dyslexia Test - IDT (Capovilla e cols., 2001; Smythe \& Capellini, 2008; Smythe \& Everatt, 2000). Foram realizadas comparações estatísticas a partir de três testes distintos: teste $t$ de student para amostras independentes, One-way ANOVA e teste post-hoc de Tukey. $O$ teste $t$ foi aplicado para comparar os grupos com e sem dificuldades de leitura. O teste de ANOVA verificou se existem diferenças estatisticamente significativas entre os subgrupos. A análise post-hoc pelo teste de Tukey avaliou quais dos subgrupos apresentaram diferenças estatisticamente significativas entre si. Por fim, o desempenho em cálculos aritméticos básicos foi correlacionado com o desempenho nas tarefas neuropsicológicas e a inteligência, através do teste de Correlação de Pearson. A linguagem oral foi avaliada pela compreensão oral de história ouvida (Salles, 2005) e a inteligência pelo Teste de Matrizes Progressivas Coloridas de Raven (Angelini, Alves, Custódio, Duarte \& Duarte 1999).

\section{Resultados}

O grupo competente em leitura/escrita apresentou desempenho médio de 6,4 ( $D P=2,2)$ em cálculos matemáticos e o grupo com dificuldade apresentou escore médio de 5,08 $(\mathrm{DP}=1,89)$. Conforme a comparação de médias entre os grupos (teste $\mathrm{T}$ de Student para amostras independentes), as diferenças não foram estatisticamente significativas. De acordo com os resultados no subteste de cálculos aritméticos do IDT (Capovilla e cols., 2001; Smythe \& Cappellini, 2008; Smythe \& Everatt, 2000) e variabilidade intragrupo, cada um dos dois grupos de crianças, competente em leitura e escrita e com dificuldades de leitura e escrita, foi subdividido em dois subgrupos conforme o desempenho em cálculos aritméticos, dando origem a quatro subgrupos, a saber: subgrupo 1 - competente em leitura e escrita e competente na aritmética (CLE/CA); subgrupo 2 - competente em leitura e escrita e com dificuldade na aritmética (CLE/DA); subgrupo 3 - com dificuldade de leitura e escrita, competentes na aritmética (DLE/CM) subgrupo 4- com dificuldade de leitura e escrita e com dificuldade na aritmética DLE/DA. O critério para separação dos grupos em subgrupos em termos de escores em cálculos aritméticos foi de escores até $50 \%$ de acertos enquadrados na denominação "com dificuldades em cálculos aritméticos" e os demais escores nos subgrupos "competentes em cálculos aritméticos".

Conforme a Tabela 1, na aritmética, a média do subgrupo 1 (CLM/CA) foi 7,70, enquanto que a do subgrupo 2 (CLE/DA) foi 4,20. Inversamente, a média do subgrupo 3 (DLE/CA) foi 6,33 , enquanto que a média do subgrupo 4 (DLE/DA ) foi 4,80. O teste de ANOVA one-way comparou as médias entre os subgrupos na aritmética e apontou uma diferença estatisticamente significativa ao nível de $5 \%$ de significância $(F(3,22)=20 \cdot 170 ; p<0,05)$. Conforme a análise post-hoc (teste de Tukey), os subgrupos 1 e 3 não se diferenciaram entre si e os subgrupos 2 e 4 também não se diferenciaram entre si em relação ao desempenho aritmético. Foi encontrada uma diferença estatisticamente significativa $(p<0,05)$ entre os subgrupos 2 e 3.

Desta forma, os considerados com dificuldade na aritmética efetivamente se diferenciam daqueles considerados competentes nesta área, independente de suas habilidades de leitura e escrita. Assim, entre as crianças competentes em leitura e escrita, algumas apresentam dificuldade na aritmética (subgrupo 2). De modo inverso, algumas crianças com dificuldade em leitura e escrita apresentam-se competentes em relação à aritmética (subgrupo 3).

A Tabela 1 apresenta o desempenho em aritmética e nas demais funções neuropsicológicas avaliadas dos subgrupos de crianças conforme desempenhos em leitura e em cálculos aritméticos (grupos competente em leitura e escrita - com e sem dificuldade na aritmética - e grupos com dificuldade em leitura e escrita - com e sem dificuldade na aritmética).

Os alunos do subgrupo 1 (CLE/CA) evidenciaram competências na aritmética básica, efetuando corretamente a maior parte das operações. Já o subgrupo 2 (CLE/DA), também competente nas tarefas de leitura e escrita, apresentou problemas até mesmo nas tarefas aritméticas mais elementares, que demandavam somente adições de números com um algarismo. As crianças do subgrupo 2 (CLE/DA) não apresentaram diferenças em relação ao grupo 1 (CLE/ CA) quanto a competências em outros processos cognitivos avaliados. No entanto, mostraram dificuldades na recupe- 
Tabela 1. Médias e desvio-padrão de cada subgrupo em matemática, no Teste Raven e nas demais funções neuropsicológicas.

\begin{tabular}{|c|c|c|c|c|c|c|c|c|}
\hline \multirow{3}{*}{ Funções/tarefas } & \multicolumn{4}{|c|}{$\begin{array}{l}\text { Grupo competente em } \\
\text { leitura e escrita } \\
(n=15)\end{array}$} & \multicolumn{4}{|c|}{$\begin{array}{l}\text { Grupo com dificuldade em } \\
\text { leitura e escrita } \\
(n=12)\end{array}$} \\
\hline & \multicolumn{2}{|c|}{$\begin{array}{l}\text { CM } \\
\text { Grupo } 1 \\
(n=10)\end{array}$} & \multicolumn{2}{|c|}{$\begin{array}{l}\text { DM } \\
\text { Grupo } 2 \\
(n=5)\end{array}$} & \multicolumn{2}{|c|}{$\begin{array}{l}\text { CM } \\
\text { Grupo } 3 \\
(n=7)\end{array}$} & \multicolumn{2}{|c|}{$\begin{array}{l}\text { DM } \\
\text { Grupo } 4 \\
(n=5)\end{array}$} \\
\hline & $M$ & $\mathrm{DP}$ & $M$ & $\mathrm{DP}$ & M & DP & $M$ & DP \\
\hline Matemática & 7,70 & 1,25 & 4,20 & 0,83 & 6,33 & 0,51 & 4,80 & 0,44 \\
\hline Teste Raven & 78,40 & 20,57 & 80,80 & 16,45 & 50,00 & 17,88 & 54,00 & 23,02 \\
\hline Percepto-motor & 3,86 & 0,47 & 3,53 & 0,44 & 3,14 & 0,37 & 3,33 & 0,55 \\
\hline Linguagem oral & 4,23 & 0,75 & 3,86 & 1,09 & 3,14 & 0,65 & 3,61 & 1,14 \\
\hline VP & 7,44 & 0,86 & 8,36 & 1,87 & 7,71 & 1,38 & 8,60 & 2,42 \\
\hline $\mathrm{CF}$ & 4,50 & 0,40 & 4,35 & 0,37 & 3,42 & 0,92 & 2,00 & 1,04 \\
\hline Memória verbal & 3,07 & 0,51 & 3,05 & 0,44 & 2,53 & 0,46 & 2,80 & 0,62 \\
\hline Memória NV & 3,95 & 0,49 & 3,70 & 0,57 & 3,57 & 0,53 & 3,66 & 0,81 \\
\hline
\end{tabular}

Nota. $\mathrm{CM}=$ Competentes em matemática; $\mathrm{DM}=$ Dificuldades em matemática; $\mathrm{VP}=$ Velocidade de processamento; $\mathrm{CF}=$ Consciência fonológica; NV = Não-verbal.

ração de fatos básicos e cometeram erros nas somas e subtrações elementares. Os alunos do subgrupo 3 (DLE/CA) resolveram boa parte das questões aritméticas, diferenciando-se do subgrupo 4 (DLE/DA), no qual foram constatadas dificuldades nas duas áreas avaliadas - linguagem escrita e aritmética.

$\mathrm{Na}$ análise das correlações, foram encontradas correlações estatisticamente significativas entre o desempenho em cálculos aritméticos e leitura de palavras isoladas $(r=0,42 ; p<0,05)$, e três medidas de consciência fonológica, a saber rima $(r=0,4 ; p<0,05)$, aliteração $(r=0,47 ; p<0,05)$ e exclusão fonêmica $(r=0,41 ; p<0,05)$. As demais análises não mostraram significância estatística.

\section{Discussão}

O presente estudo corrobora os dados da literatura sobre a variabilidade dos perfis funcionais em termos de habilidades aritméticas em crianças com e sem dificuldades em leitura e escrita (Fuchs \& Fuchs, 2002; Jordan, 2007). Tal resultado sugere que existem habilidades cognitivas comuns à leitura e escrita e à aritmética, como, por exemplo, significado das palavras numéricas, elaboração de conceitos e compreensão leitora de problemas verbais. Os subgrupos que apresentaram dificuldades em ambas habilidades (linguagem escrita e habilidades aritméticas) dão suporte a estas questões.

Atualmente, há consenso entre os pesquisadores de que grande parte das crianças com dificuldades na arit- mética também apresentam dificuldades na leitura. Isso é compreensível, considerando-se o papel da linguagem na aprendizagem escolar e que um déficit nuclear da linguagem, como o processamento fonológico, pode atingir tanto a aprendizagem da leitura e da escrita quanto da aritmética (Dowker, 2004; Durant, Hulme, Larkin \& Snowling, 2005; Fuchs e cols. 2005; Fuchs e cols., 2006; Geary, 2004; Geary e cols., 2000; Jordan, 2007; Jordan \& Hanich, 2003).

Porém, por outro lado, há crianças que apresentam desempenho adequado em um dos domínios e deficitário no outro. Há habilidades predominantemente numéricas, por exemplo, como o senso numérico e a construção de uma linha numérica mental. Os subgrupos que apresentaram desempenho competente em leitura/escrita, mas deficitário em habilidades aritméticas, e o subgrupo com perfil oposto (com dificuldade de leitura e escrita e competente nas habilidades aritméticas) corroboram tal constatação. Para Geary e Hoard (2005), crianças competentes em leitura e escrita e com dificuldade na aritmética têm uma fragilidade no senso numérico e, consequentemente, no armazenamento e recuperação de fatos numéricos. Esta característica foi evidenciada nas crianças do subgrupo 2 (CLE/DA) do presente estudo. Tais diferenças no desempenho aritmético e nas habilidades de leitura e escrita, no âmbito da neurociência cognitiva, são denominadas dissociações duplas (Gazzaniga, Ivry \& Mangun, 2006) e são muito elucidativas no estudo dos processos cognitivos (Fuchs e cols., 2006; Jordan, 2007), na medida em que esclarecem a interdependência e independência entre as funções cognitivas. 
O desempenho do subgrupo 3 (DLE/CA) vai ao encontro dos achados recentes de que nem todas as crianças com problemas de linguagem escrita apresentam déficits na aritmética. Nesta pesquisa, as crianças do subgrupo 3 (DLE/ CA) apresentaram melhor desempenho aritmético do que as do subgrupo 2 (CLE/DA). Segundo os trabalhos de Jordan e cols. (2002), as crianças competentes em matemática são mais rápidas na recuperação dos fatos básicos e, consequentemente, são mais fluentes em cálculo do que as crianças que apresentam o padrão inverso. Como evidenciam os trabalhos de Fuchs e Fuchs (2002), corroborando os achados de Jordan, no presente estudo, as crianças do subgrupo 3 (DLE/CA) assemelharam-se às do subgrupo 1 (CLE/CA) no senso numérico e na fluência de cálculos, mas provavelmente se diferenciariam nas tarefas em que a competência linguística é fundamental, como na resolução de problemas apresentados verbalmente e na elaboração de conceitos (não avaliados neste estudo). Dificuldades moderadas de processamento fonológico podem apresentar impedimentos para aprender a ler e não apresentar nenhum obstáculo no sistema numérico (Robinson, Menchetti \& Torgesen, 2002).

Considerando as correlações entre desempenho aritmético e os processos neuropsicológicos, nesta pesquisa evidenciaram-se correlações estatisticamente significativas entre desempenho aritmético, leitura e consciência fonológica apenas. Ressalta-se que foi examinada a competência em cálculos, sem exigência de leitura e interpretação como nos problemas verbais, e, mesmo assim, encontrou-se correlação estatisticamente significativa entre consciência fonológica e habilidades matemáticas.

\section{Considerações finais}

Tanto crianças enquadradas como competentes em leitura e escrita como aquelas consideradas com dificuldades nestas habilidades podem variar em termos de desempenho em habilidades aritméticas. Algumas delas podem ter dificuldades mais amplas, que incidem sobre a linguagem e escrita e as habilidades aritméticas (associações de sintomas), enquanto outras podem apresentar dificuldades específicas em um ou outro domínio (dissociações de sintomas). Este estudo traz uma importante contribuição para o campo da aprendizagem e das dificuldades de aprendizagem, esclarecendo sobre as associações e dissociações entre os processos cognitivos relacionados com a leitura e escrita e com a aritmética. Das 27 crianças examinadas, 52\% evidenciaram associações entre os desempenhos nas habilidades de leitura e escrita e da matemática. Um percentual considerável - 48\% - revelou dissociações entre os desempenhos nas tarefas que avaliam habilidades linguísticas (leitura e escrita) e numéricas. Apesar de haver dissociações, os escores em habilidades aritméticas estavam correlacionados aos de leitura e de consciência fonológica, sugerindo que habilidades básicas podem estar subjacentes aos domínios linguísticos e aritméticos.
Mais investigações são necessárias para que seja possível avançar no entendimento das dificuldades na aprendizagem da matemática, associadas ou não às alterações de linguagem escrita, assim como no planejamento e execução de programas preventivos e terapêuticos no processo inicial da escolarização. As experiências matemáticas escolares iniciais são decisivas para o desenvolvimento numérico posterior. No Brasil, urge intensificar os cuidados preventivos das crianças, pelo menos a partir dos 4 ou 5 anos, através de programas de Educação Infantil que contemplem as construções e o processamento numérico e os diferentes tipos de memória - semântica, fonológica e visuoespacial. De acordo com as pesquisas, os fundamentos cognitivos do pensamento matemático desenvolvem-se dos 2 aos 6 anos através da interação com adultos e outras crianças. Daí a importância de programas educacionais que universalizem a Educação Infantil, com propostas que visem ao enriquecimento da experiência linguística, numérica e social.

É igualmente urgente reformular as práticas escolares que enfatizam os procedimentos, especialmente de cálculos, em detrimento das habilidades conceituais que os fundamentam. Como alerta Geary (2004), competências conceituais e procedurais andam juntas, reforçando-se mutuamente. As crianças provindas de níveis socioculturais favorecedores desenvolvem as bases perceptivas e conceituais do conhecimento numérico, tanto dentro da escola, quanto fora dela. Portanto, dependem menos da escola para seu desenvolvimento perceptivo, linguístico e conceitual. No caso de crianças desfavorecidas socioculturalmente, cabe à escola prover essas experiências de vida que sustentam a aprendizagem escolar.

\section{Referências}

Angelini, A. L., Alves, I. C. B., Custódio, E. M., Duarte, W. F., \& Duarte, J. L. M. (1999). Matrizes Progressivas Coloridas de RAVEN Escala especial. São Paulo: Centro Editor de Testes e Pesquisas em Psicologia.

Baddeley, A. D. (1996). Exploring the central executive. The Quarterly Journal of Experimental Psychology, 49(1), 5-28.

Baddeley, A. D. (2000). The episodic buffer: A new component of working memory? Trends in Cognitive Sciences, 4(11), 417-423.

Baddeley, A. D., \& Andrade, J. (2000). Working memory and the vividness of imagery. Journal of Experimental Psychology General, 129(1), 126-145.

Berch, D. B., \& Mazzocco, M. M. M. (2007). Why is math so hard for some children? The nature and origins of mathematical learning difficulties and disabilities. Baltimore: Brookes Publishing Company. 
Brandão, A. C. P., \& Spinillo, A. G. (2001). Produção e compreensão de textos em uma perspectiva de desenvolvimento. Estudos de Psicologia, 6(1), 51-62.

Capovilla, F. C., Smythe, I., Capovilla, A. G., \& Everatt, J. (2001). Adaptação brasileira do International Dyslexia Test: Perfil cognitivo de crianças com escrita pobre. Temas sobre Desenvolvimento, 10(57), 30-37.

Costa, A. C. (2007). Estratégias de ensino para crianças e adolescentes com transtorno de déficit de atenção/hiperatividade (TDAH): Possibilidades de intervenção pedagógica na aritmética. Projeto de Tese, Universidade Federal do Rio Grande do Sul, Porto Alegre-RS.

Dehane, S., \& Cohen, L. (1995). Towards an anatomical and functional model of number processing. Mathematical Cognition, 1(1), 83120.

Delors, J. (1999). Educação: Um tesouro a descobrir; Relatório para a UNESCO da Comissão Internacional sobre educação para o século XXI. São Paulo: Cortez Editora e UNESCO Brasil.

Dowker, A. D. (2004). What works for children with mathematical difficulties?. Londres: University of Oxford.

Durant, M., Hulme, C., Larkin, R., \& Snowling, M. (2005). The cognitive foundations of reading and arithmetic skills in 7- to 10-year-olds. Journal of Experimental Child Psychology, 91(2), 113-136.

Fuchs, L. S., Compton, D. L., Fuchs, D., Paulsen, K., Bryant, J. D., \& Hamlett, C. L. (2005). The prevention, identification and cognitive determinants of math difficulties. Journal of Educational Psychology, 97(3), 493-513.

Fuchs, L. S., \& Fuchs, D. (2002). Mathematical problem solving profiles of students with mathematics disabilities with and without co-morbid reading disabilities. Journal of Learning Disabilities, 35(6), 563-573.

Fuchs, L. S., Fuchs, D., Compton, S. R., Powell, P. M., Seethaler, A. M., Capizzi, C., Schatschneider, C., \& Fletcher, J. M. (2006). The cognitive correlates of third-grade skill in arithmetic, algorithmic computation and arithmetic word problem. Journal of Educational Psychology, 98(1), 29-43.

Gazzaniga, M., Ivry, R., \& Mangun, G. (2006). Neurociência cognitiva: a biologia da mente. Porto Alegre: Artmed.

Geary, D. C. (1993). Mathematical disabilities: Cognitive, neuropsychological and genetics components. Psychological Bulletin, 114(2), 345-362.

Geary, D. C. (2004). Mathematics and learning disabilities. Journal of Learning Disabilities, 37(1), 4-15.
Geary, D. C. (2006). Development of mathematical understanding. Em D. Kuhl \& R. S. Siegler (Orgs.), Cognition, perception and language - Handbook of child psychology (Vol. 2, pp. 777-810). New York: John Wiley \& Sons.

Geary, D. (2007). An evolutionary perspective on learning disabilities in Mathematics. Developmental Neuropsychology, 32(1), 471519.

Geary, D. C., Hamson, C. O., \& Hoard, M. K. (2000). Numerical and arithmetical cognition: A longitudinal study of process and concept deficits in children with learning disability. Journal of Experimental Child Psychology, 77(3), 236-263.

Geary, D. C., \& Hoard, M. K. (2005). Learning disabilities in arithmetic and mathematics: Theoretical and empirical perspectives. Em J. I. D. Campbell (Org.), Handbook of mathematical cognition (pp. 253267). New York: Psychological Press.

Gersten, R., Clarke, B., \& Mazzocco, M. M. M. (2007). Historical and contemporary perspectives on mathematical learning disabilities. Em D. Berch \& M. M. M. Mazzocco (Orgs.), Why is math so hard for some children? The nature and the origins of mathematical learning difficulties (pp. 7-28). Baltimore: Brookes Publishing Company.

Jordan, N. C. (2007). Do words count? Em D. B. Berch \& M. M. M. Mazzoco (Orgs.), Why is math so hard to some children? The nature and the origins of mathematical learning difficulties ( $\mathrm{pp}$ 107-120). Baltimore: Brookes Publishing Company.

Jordan, N. C., \& Hanich, L. (2003). Characteristics of children with moderate mathematics deficiencies: A longitudinal perspective. Learning Disabilities, Research and Practice, 18(4), 213-221.

Jordan, N. C., Hanich, L., \& Kaplan, D. (2003). A longitudinal study of mathematical competencies in children with specific mathematics difficulties versus children wit co-morbid mathematical and reading difficulties. Child Development, 74(3), 834-850.

Jordan, N. C., Kaplan, D., \& Hanich, L. B. (2002). Achievement growth in children with learning disabilities in mathematics: Findings of a two-year longitudinal study. Journal of Educational Psychology, 94(3), 586-597.

Kleiman, A. (2001). Leitura: ensino e pesquisa. Campinas, SP: Pontes.

Lins e Silva, M. E., \& Spinillo, A. G. (1998). Uma análise comparativa da escrita de histórias pelos alunos de escolas públicas e particulares. Revista Brasileira de Estudos Pedagógicos, 79(193), 5-16.

Lins e Silva, M. E., \& Spinillo, A. G. (2000). A influência de diferentes situações de produção na escrita de histórias. Psicologia: Reflexão e Crítica, 13(3), p. 337-350. 
Mazzocco, M. M. M. (2007). Defining and differentiating mathematical learning disabilities and difficulties. Em D. Berch \& M. M. M. Mazzocco (Orgs.), Why is math so hard for some children? The nature and the origins of mathematical learning difficulties (pp. 2947). Baltimore: Brookes Publishing Company.

Moojen, S., \& Costa, A. C. (2006). Semiologia psicopedagógica. Em N. T. Rotta, L. Ohlweiler \& R. S. Riesgo (Orgs.), Transtornos da aprendizagem - Abordagem neurobiológica e multidisciplinar (pp. 103-112). Porto Alegre: Artmed.

Orrantia, J. (2006). Dificultades en el aprendizaje de las matemáticas: Uma perspectiva evolutiva. Revista Psicopedagogia, 71, 150-180.

Pinheiro, A. M. V. (2001). Heterogeneidade entre leitores julgados competentes pelas professoras. Psicologia: Reflexão e Crítica, 14(3), 537-551.

Robinson, C. S., Menchetti, B. M., \& Torgesen, J. K. (2002). Toward a two-factor theory of one type of mathematics disabilities. Learning disabilities Research \& Practice, 17(2), 81-89.

Salles, J. F. (2001). O uso das rotas de leitura fonológica e lexical em escolares: relações com compreensão, tempo de leitura e consciência fonológica. Dissertação de Mestrado não publicada. Curso de Pós-Graduação em Psicologia do Desenvolvimento, Universidade Federal do Rio Grande do Sul - UFRGS, Porto Alegre, RS.

Salles, J. (2005). O uso das rotas de leitura fonológica e lexical em escolares: relações com compreensão, tempo de leitura e consciência fonológica. Dissertação de Mestrado, Universidade Federal do Rio Grande do Sul, Porto Alegre-RS.
Salles, J. F., \& Parente, M. A. M. P. (2002a). Processos cognitivos na leitura de palavras em crianças: Relações com compreensão e tempo de leitura. Psicologia: Reflexão e Crítica, 15(2), 321-331.

Salles, J. F., \& Parente, M. A. M. P. (2002b). Relações entre os processos cognitivos envolvidos na leitura de palavras e as habilidades de consciência fonológica em escolares. Pró-Fono Revista de Atualização Científica, 14(2), 175-186.

Salles, J. F., \& Parente, M. A. M. P. (2006). Heterogeneidade nas estratégias de leitura/escrita em crianças com dificuldade de leitura e escrita. Psico, 37(1), 83-90.

Salles, J. F., \& Parente, M. A. M. P. (2007). Avaliação da leitura e escrita de palavras em crianças de $2^{\mathrm{a}}$ série: abordagem neuropsicológica cognitiva. Psicologia: Reflexão e Crítica, 20(2), 218-226.

Salles, J. F., \& Parente, M. A. M. P. (2008). Variabilidade no desempenho em tarefas neuropsicológicas entre crianças de $2^{a}$ série com dificuldades de leitura e escrita. Arquivos Brasileiros de Psicologia, 60(1), 32-44.

Smythe, I., \& Capellini, S. A. (2008). Protocolo de avaliação de habilidades cognitivo-linguísticas. Marília, SP: Fundepe.

Smythe, I., \& Everatt, J. (2000). Dyslexia diagnosis in different languages. Em L. Peer \& G. Reid (Orgs.), Multilingualism, literacy and dyslexia (pp. 12-21). London: David Fulton Publishers.

Spinillo, A. G. (2001). A produção de histórias por crianças: a textualidade em foco. Em J. Correa, A. Spinillo \& S. Leitão (Orgs.). Desenvolvimento da linguagem: escrita e produção textual (pp. 73-116). Rio de Janeiro: NAU Editora: FAPERJ.

\section{Sobre as Autoras}

Clarissa Seligman Golbert (mcgolbert@uol.com.br)

Universidade Federal do Rio Grande do Sul, Porto Alegre - RS

Jerusa Fumagalli de Salles (jerusafs@yahoo.com.br)

Universidade Federal do Rio Grande do Sul, Porto Alegre - RS

Recebido em: 05/06/2009 Reformulado em: 29/07/2010 (19)

Reformulado em: 07/10/2010 (2a)

Aprovado em: 28/10/2010

\section{Correspondência}

Clarissa Seligman Golbert

Rua Cauduro, 66 apto 1101 Bairro Bom Fim, 90035-110 Porto Alegre RS 